\title{
Vulvar Pigmented Growth: A Psychological Concern?
}

\author{
Deeptara Pathak ', Sudha Agrawal ${ }^{2}$, Tapan Dhali ${ }^{2}$ \\ Delhi Dermatology Group Kubba, New Delhi, India' \\ Dept of Dermatology, B. P. Koirala Institute of health sciences, Nepal'
}

\begin{abstract}
Vular nevi are uncommon. We report a case of an 18 year old girl presented to us as a pigmented growth on vulva since birth. The lesion was small at the time of birth but gradually increased with time. On clinical examination there was a pigmented lobulated growth of vulva of size of $5 \mathrm{~cm} \times 8 \mathrm{~cm}$. A punch biopsy from the growth was done which was consistent with congenital intradermal nevus. Patient was referred to gynecology department for resection of the lesion. We are reporting this case for its rarity and to initiate awareness among clinicians the importance of clinico-pathological evaluation and managing such patient to have a normal sexual life and to decrease any psychological sequelae associated with any vulvar problem.
\end{abstract}

Key words - Congenital nevus, Vulvar nevi, atypical nevi

\section{Introduction}

The prevalence of the lower genital tract diseases seen in dermatology and gynecology clinics is unknown. Although the vulva may be involved in several dermatological disorders, there are some, such as lichen sclerosus and lichen planus that have a predilection for vulvar skin ${ }^{1}$. Melanocytic nevi are very common on skin surface but rare in the vulvar area.

\section{Case}

An 18 year old girl presented to us as a pigmented growth on vulva since birth. The lesion was small at the time of birth but gradually increased with time. On clinical examination there was a lobulated growth of size of $5 \mathrm{~cm} \times 8 \mathrm{~m}$ involving right labia majora, bilateral labia minora, clitoris and a part of left labia minora extending to involve a part of mons pubis as shown in Figure 1. It was firm to smooth in consistency, non tender. There were no secondary changes . A punch biopsy from the growth was done and showed nest of

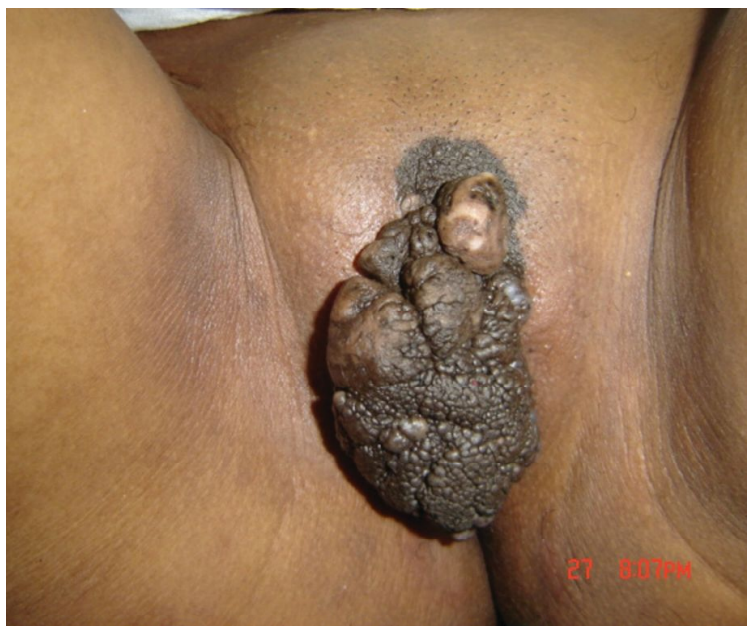

Fig 1. Congenital nevus of vulva, as lobulated pigmented growth involving right labia majora, bilateral labia minora, clitoris and a part of left labia minora extending to involve a part of mons pubis.

Correspondence

Dr Deeptara Pathak,

Consultant Dermatologist,

National Institute of Neurological and allied sciences,

Bansbari,Kathmandu

Ph: 00919868790634 ; 00911124196666

E-mail: drdeeptarapathak@yahoo.com

Fax: 00911124196677 


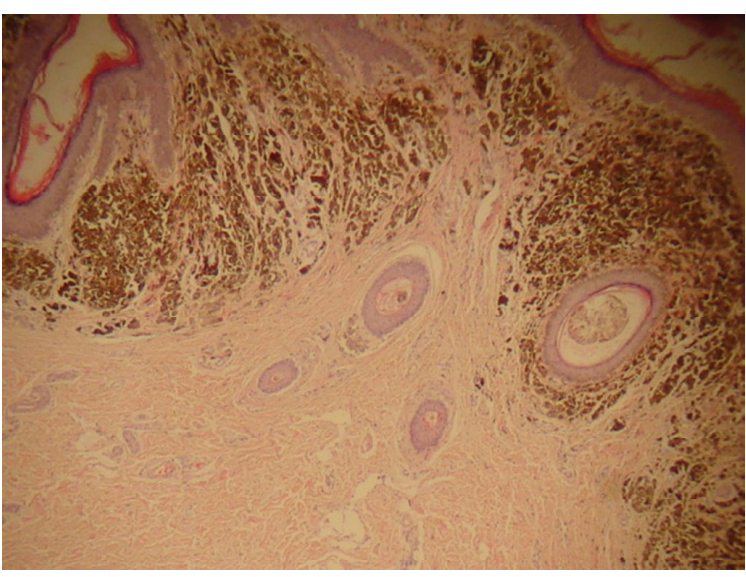

Fig 2. A punch biopsy from the vulvar growth, showing nest of nevus cells involving papillary and reticular dermis also around appendages which was consistent with congenital intradermal nevus. ( $10 \times$ magnification)

nevus cells involving papillary and reticular dermis also around appendages which was consistent with congenital intradermal nevus as shown in Figure 2. Patient was referred to gynecology department for resection of the lesion.

\section{Comment}

The term "vulva" is derived from the Latin word for "covering" and was originally used to describe the uterus ${ }^{2}$. The most commonly encountered vulvar dermatomes are papulosqamous disorders, which include psoriasis, seborrhea and the lichens (lichen planus, lichen sclerosus, lichen simplex chronicus) and chronic or recurrent infections ${ }^{2-3}$. Melanocytic nevi are very common on skin surface but rare in the vulvar area. Nevi are hamartomas of melanocyte derived from neural crest. There are several clinical types of nevi like flat nevi, slightly elevated nevi, papillomatous nevi, dome shaped nevi, pedunculated nevi, dysplastic nevi, giant congenital nevomelanocytic nevi. The patterns can be found anywhere in the vulva. Benign nevi are uniformly pigmented, well circumscribed, and usually under $6 \mathrm{~mm}$ in diameter ${ }^{4}$. Vulvar nevi are uncommon in gynecological practice with a prevalence of $2.3 \%{ }^{5}$. Christensen et al did a comparative histological study of vulvar and common nevi and found that most of the vulvar lesions were unremarkable ${ }^{6}$. The term atypical melanocytic nevi of the genital type (AMNGT) are the nevi seen on the vulva of premenopausal women, which are clinically unremarkable nevi and present with histologic features that simulate some features of melanoma and usually have a benign clinical course $^{7,8}$. These lesions have possibility of diagnostic error but clinically unremarkable. We report a case of 18year old girl who presented to us as a pigmented growth on vulva since birth. The lesion was small at the time of birth but gradually increased to a size of $5 \mathrm{~cm} \times 8 \mathrm{~cm}$ involving right labia majora, bilateral labia minora, clitoris and a part of left labia minora extending to involve a part of mons pubis. A punch biopsy from the growth was consistent with congenital intradermal nevus. Patient was referred to gynecology department for resection of the lesion. Any lesion or chronic symptoms relating to vulva leads to women curiosity of being having malignant tumor or HIV infection and subsequently causes more social, mental and psychological disharmony. Most women have no anatomical education and when they have genital cutaneous problems, they find it hard to deal with the problem in an unfamiliar area that they cannot see. Because of the sensitive nature of these disorders, women often suffer in silence and do not seek medical care. We are reporting this case for its rarity and as an awareness among treating physician in terms of clinicohistopathological evaluation of such patient and management so that it helps such patients to come up from the embarrassment associated with its symptoms and the psychological sequelae that stem from its interference in normal sexual relations.

\section{References}

1. Lewis FM. Vulval lichen planus. Br J Dermatol 1998; 138(4): 569-575.

2. Lewis FM. Vulval Disease from the 1800 s to the new millennium. J Cutan Med Surg 2002; 6(4):340-344.

3. McKay M. Vulvar dermatoses: common problems in dermatological \& gynaecological practice. Br J Clin Pract Suppl 1990; 71: 5-10.

4. Fisher BK, Margesson LJ. The vulva, In: Fisher BK, Margesson LJ (Eds). Genital skin disorders, $1^{\text {st }}$ edition. Philadelphia: Mosby 1998; 99-196.

5. Rock B, Hood AF, Rock JA. Prospective study of vulvar nevi. J Am Acad Dermatol 1990; 22:104106

6. Christensen WN, Friedman KF, Woodruff JD, et al. Histologic characteristics of vulvar nevocellular nevi. J Cutan Pathol 1987; 14:8791

7. Friedman RJ, Ackerman AB. Difficulties in the histologic diagnosis of melanocytic nevi on the vulvae of premenopausal women. In: Ackerman $\mathrm{AB}$, ed., Pathology of malignant melanoma. New York: Masson 1981:119-127.

8. Ribe A. Melanocytic lesions of the genital area with attention given to atypical genital nevi. J Cutan Pathol 2008 Nov; 35 Suppl 2:24-7. 